discharge. It is claimed with some justice that aspiration of an infective fluid causes a flow of serum rich in opsonins into the space evacuated, and also that Bier and Klapp's cupping glasses used in connexion with sinuses have a similar effect. But out of a series of papers on the opsonic treatment which has lately appeared ${ }^{6 t}$ little light has been thrown on the application of it to acute conditions, and much wider experience is necessary before a definite judgment can be pronounced.

Bibliography.-1. Oettinger: Charcot's Traité de Médecine, vol. $\nabla$. p. 533. 2. Widal : Brouardel's Traité de Médecine, vol. iii., p. 774 . 3. Allchin : Allbutt's System of Medicine, vol. iii., p. 632 . 4. Morrow : New Sydenham Society, vol. cxliii. 5. Poncet: Gazette Hebdomaraire, 1874, p. 133; Schlange: Archiv fur Klinische Chirurgie, vol. xxxvi. 6. Mantle: Brit. Med. Jour., 1887, vol, i., p. 1381. 7. Widal : Loc. cit. 8. Schuchardt : Billroth and Lueke's Deutsche Chirurgie, vol.xxviii. (A) p. 292. 9. Church: Allbutt's System of Medicine, vol. iii. 10. Leyden Medical Society, Berlin, 1894 ; Beattie : Brit. Med. Jour., 1906, vol. ii. p. 1781. 11. Cheadle: Allbutt's System of Medicine, vol. iii., p. 47 p. 505 14 T : Loc. cit. 13. Chassaignac : Gazette Médical, 18. 15. Volk . S . Driessen, Zentralblatt fur Chirurgie, 1880, p. 673 . 16. Schede: Mittheilungen aus der Berliner stäntischen Krankenhauses in Friedrichshain, 1878. 17. Müller: Deutsche Zeitschrift fúr Chirugie, vol. xxi. 18. Lexer: Archiv für Klinische Chirurgie, vol. lvii., p. 879 . 19. T. P. Pick: THE LANCET, 1894, vol, i., p. 1543; Cautley: vol. x., p. 241. 20. Von Brung and Honsell: Ibid., vol. xxiv., p. 4l. 21. Guyot: Revue de Chirurgie, vol. xxix., p. 271.22. Lannelongue: Bulletins et Mémoires de la Société de Chirurgie,
vol, xiii.: p 17. 23. Macnamara: Brit. Med. Jour., 1887, vol. 1i., p. 13;

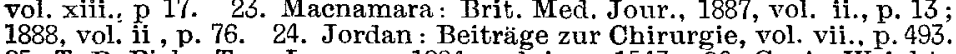
25. T. P. Pick: THE LANCET, 1894, vol. i., p. 1543. 26. G. A. Wright: THE LANCET, 1881, vol. ii., p. 127. 27. Bergmann, Bruns, and MHE LANCET, 1881, vol. ii., p. 127. 27. Bergmann, Bruns, and Mikulicz's Practische Chirurgie. 28. Bardenheuer: Archiv für
Klinische Chirurgie, vol. xli. 29. Widal : Loc. cit. 30. Kraske : Archiv Klinische Chirurgie, vol. xli. 29. Widal : Loc. cit. 30. Kraske : Archiv
für Klinische Chirurgie, vol, xxxiv. 31. Pick : Loc. cit. 32. Bloodgood: fuir Klinische Chirurgie, vol, xxxiv. 31. Pick : Loc. cit. 32. Bloodgood: Progressive Medicine, 1904, vol. iv., p. 205. 33. Lexer: Archiv fur
Klinische Chirurgie, vol. lxxi., p. 9; vol. lxxiii. p. 481. 34. Bloodgood: Klinische Chirurgie, vol. lxxi., p. 9; vol. lxxiii., p. 481. 34. Bloodgood: Medicinische Wochenschrift, 1896, p. 751. 37. Cave: THE LANCET, 1901, Medicinische Wochenschrift, 1896, p. 751. 37. Cave: THE LANCET, 1901,
vol. i. p. 82. 38. Pasteur and Courtland: THE LANCET, 1906, vol, vol. 1., p. 82. 38. Pasteur and Courtland: THE LANCET, 1906, vol. i.,
p. 1747. 39. Griffon: Bulletin de la Société d'Anatomie, 1896. 40. p. 1747. 39. Griffon: Bulletin de la Société d'Anatomie, 1896. 40 1896. 41. Chvostek: Verhandlungen des XV. Kongresses fir inner Medicin. 42. Hofbauer: Mittheilungen aus der Grenzgebieten der Medicin und Chirurgie, vol. iji., p. 71. 43. Flint: Annals of Surgery, 1905, p. 570. 44. William Hunter: Practitioner, 1900 45. Courderoy: Thèse de Paris, 1900; Raffin: Thèse de Bordeaux, 1900. 46. Rogers: THE LANCET, 1905, vol i., p. 501; LANCET, 1903, vol. ii., p. 161; Franke: Mitheilungen H. Marsh : Brit Med der Medicin und Chirurgie, vol. v., p. 321 Ostéomyélite de Croissance." 48. Braquehaye : Gazette Hebdomadaire de Médecine et Chirurgie, 1895; Koplick: American Journal of Medical Sciences, 1892. 49. Chipault : Bulletin de la Société d'Anatomie 1890 ; Lamothe: Thèse de Paris, 1898; G. A. Wright: Thr LAxceT, 1902, vol. i., p. 1376; Koplick : Loc. cit. 50. Kidd and Symes-Thompson : The Lancet, 1902, vol. i., p. 1376. 51. Braquehaye: Loc. cit.

Poynton: Medical Society O London, THE LANCET, 1900, vol.i1, p. 861 54. Brault Maladies des Pays Chauds, THE LANCES, Dec. 15th, 1900. 54. Charriezieux: Thèse de Bordeaux, 1896. 55. Lefort: Bulletin d la Sociéte de Chirurgie, 1880 . 56. Sollmann : Jahrbuch fur Kinderheilkunde und physische Erziehung, 1895. 57. Fraenkel: Mittheilungen aus der Grenzgebieten der Medicin und Chirurgie. vol. xii. p. 419. 58. Bloodgood : Loc. cit, 59. Widal: THE LANCET, 1901, vol. p. 84. 60. Hermann: Annales de l'Institut Pasteur, 1891. 61. Kasparek blatt fur Chirurgie, 1906. 63. German Surgical Congress, 1906, Zentralblatt fur Chirurgie, 1906. 63 . German Surgical Congress, 1906, ZentralMay, 1908. 65. G. B. Buchanan: THE LANCET, 1908, vol. ii., p. 1430. Glasgow.

Royal College of Surgeons in Ireland.The following is the list of prizes awarded for the winter session, 1909-10. Systematic Anatomy: D. J. Flanagan, first prize (£2) and medal ; G. E. Pepper, second prize (£1) and certificate. Descriptive Anatomy: W. I. Adams, first prize (£2) and medal; D. J. Flanagan, second prize (£1) and certificate. Practical Anatomy : First year : W. Morrow, first prize (£2) and medal; R. A. Austin, second prize (£1) and certificate. Second year: G. C. Sproule, first prize (£2) and medal ; W. I. Adams and G. E. Pepper (equal), second prize (£1) and certificate. Practice of Medicine: J. T. Duncan, first prize (£2) and medal ; T. M. Thomson, second prize (£1) and certificate. Surgery: J. T. Duncan first prize (£2) and medal; F. Warren, second prize (£1) and certificate. Midwifery: J. T. Duncan, first prize (£2) and medal; M. S. Moore, second prize (£1) and certificate. Physiology : J. D. Cherry, first prize (£2) and medal; A. F. Patterson, second prize (£1) and certificate. Chemistry: J. Lanigan, first prize (£2) and medal; L. E. Wigoder, second prize (£1) and certificate. Pathology : Vernon J. White, first prize (£2) and medal; T. T. Buckley, second prize (£1) and certificate Physics: A Merrin, first prize (£2) and medal; L. E. Wigoder, second prize (£1) and certificate. The summer session commenced on April 4th.

\section{A NEW METHOD OF TREATING ACUTE PHTHISIS BY THE ALTERNATE USE OF HUMAN AND BOVINE TUBERCULIN. ${ }^{1}$}

\author{
BY H. WARREN CROWE, M.D. OXON.
}

VACCINE therapy as practised to-day is difficult. Whether inoculations are administered by the empirical method it is difficult, or under the control of the opsonic index it is also difficult-difficult not only in the technique but also in the interpretation. In phthisis it is probably more difficult than in any other disease caused by bacterial invasion, and the more active the disease the greater this difficulty.

If we are asked wherein the difficulty chiefly lies we should be candid in replying that it lies in the avoidance of doing harm.

It is impossible to disguise the fact that tuberculin may do harm. If after a dose of tuberculin a rise of temperature occurs, accompanied by malaise, increased sputum, increased physical signs, and a disproportionately quickened pulse, and lasting for a fortnight or even longer, one cannot deny that the inoculation may have done harm. Yet I venture to suggest that this result has not infrequently followed doses of T.R. even so small as $1 / 100,000$ th milligramme. I would go further and say that in active phthisis, with a rectal temperature ranging above $100^{\circ} \mathrm{F}$, a further rise of temperature, commencing sooner or later and lasting a longer or a shorter time, is the most usual result of the smallest efficient dose of tuberculin. But if tuberculin does good in acute cases-and many will, I think, be found to bear witness to its valueand if it be granted that a rise of temperature usually results from small yet efficient doses of tuberculin, it follows that the rise of temperature is not necessarily an unfavourable symptom. Obviously the length of time that the rise is maintained is the determining factor in the result of the dose, whether it shall be for good or whether it shall be for ill

An unprejudiced observer will readily acknowledge the good effect of a dose of tuberculin if it result in a rise of temperature lasting two or three days, followed by a fall to a point below the average range preceding the dose, and if then the temperature range remains at the lower level, whereas he will undoubtedly condemn as harmful treatment an inoculation which apparently causes a rise of temperature with accompanying discomfort lasting a fortnight or longer.

Now it is quite impossible in acute phthisis to anticipate the effect of any dose with certainty (with one exception which will shortly be stated). 1/100,000th milligramme T.R may have no effect the first time, but if repeated even this minute dose may provoke a prolonged reaction. It is a usual experience when re-commencing inoculations with a minimal dose after a period of cessation, due possibly to the patient's intolerance-fancied or real-to find oneself faced at the onset by a prolonged and distressing reaction. And though it is quite conceivable that an increased vigour in the protective mechanism is the cause of the phenomenon, yet it is discouraging to the physician and terrifying to the patient and his friends. If now it were possible to devise a method whereby such an awkward reaction could be cut short tuberculin administration in acute phthisis would be shorn of half its terrors. Wright advocates a minimal dose being given if the " negative phase" be too prolonged-if, that is to say, the dose causing that phase has been too large-but this method does not always meet with success in phthisis, and if the reaction we wish to stop be caused by 1/100,000th milligramme, what conceivable dose must we employ to check it?

I have now to describe a method whereby a prolonged reaction can be cut short with almost absolute certaintyeven an obvious overdose can be prevented from doing harm and may not infrequently be turned to good account.

It is maintained by some observers that there is a very real difference between the strain of tubercle bacilli grown from the human disease and that grown from the disease as found in cattle, the typus humanus and the typus bovinus further, that human beings can be infected by either strain,

The tuberculin referred to is T. $R$. and its bovine equivalent P.T.R. (from the German "Perlsucht" = Bovine tuberculosis). 
cattle usually only by their own specific type. Further, that in using tuberoulin in the human subject an attempt should be made to determine the origin of the infecting organism and the appropriate vaccine then given. If both types are present, as is said to occur commonly in phthisis, then a mixed tuberculin should be employed. As a refinement of this it has been suggested, again, that should reactions of marked intensity follow inoculations by a tuberculin of homologous type, the alternative or heterologous vaccine may be used with advantage. Dr. Nathan Raw adrocates the "opposing vaccine" being always used, as he maintains that its curative power is greater, since it antagonises and neutralises the autogenous "tuberculin" formed
Since it is my belief, which I trust this paper will tend to justify, that the "cure" (as opposed to the "arrest") of the disease in its acute form is impossible without febrile reactions, and since the voluntary induction of a rise of temperature carries with it the responsibility for any harm which may result from such a rise being too prolonged, the great value of a reliable method for terminating at will such a "therapeutic tuberculin reaction" will be immediately apparent. I have, as far as possible, emphasised this interesting phenomenon, as upon its proper application rests the success of the method for the inoculation of acute phthisis now to be described.

A start is made by an inoculation of $1 / 100,000$ th milli-

FIG. 1.

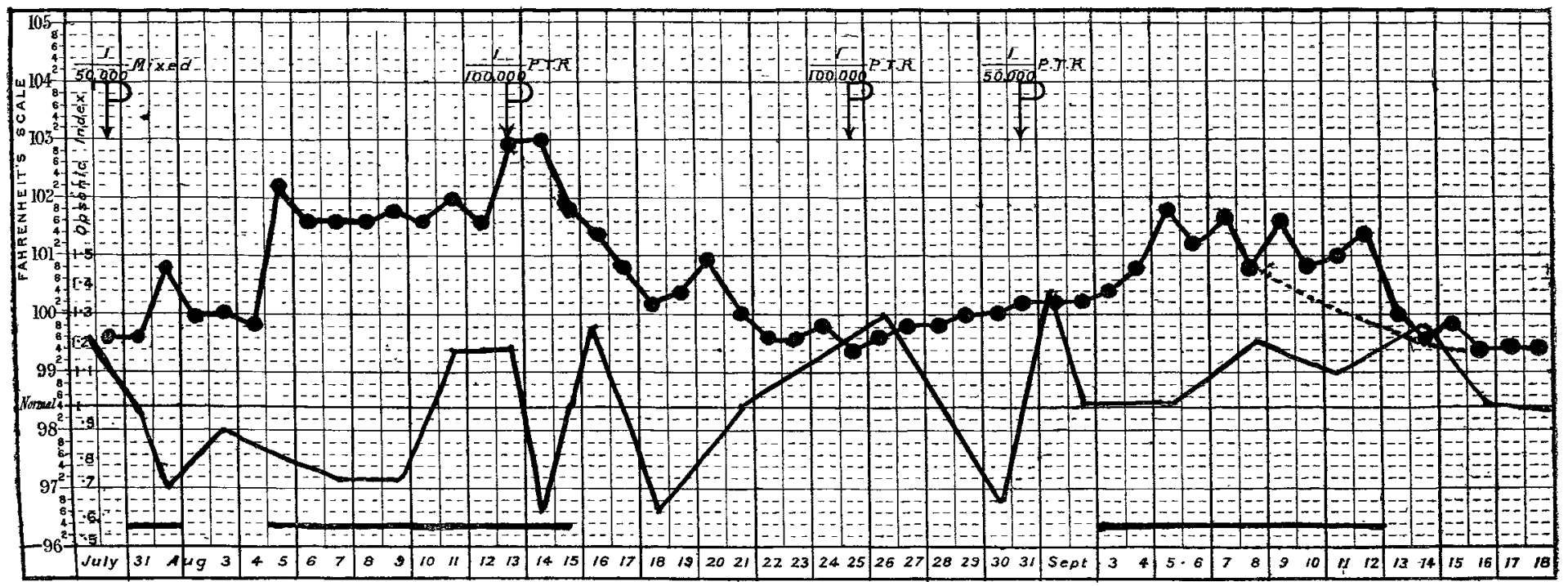

Inoculations are shown by the combined letter and arrow, the $\mathrm{T}$ in the sign indicating T.R., and the P., P.T.R. The first inoculation was a mixture of T.R. and P.T.R., hence the sign. The beaded line indicates the temperature. (In all the charts the temperature record represents the evening 9 P.M. temperature only-an excellent graphic method which I adopted after reading E. C. Hort's "Rational Immunisation in the Treatment of Pulmonary Tuberculosis.") The plain line shows the opsonic curve. Days of malaise, increased sputum, and physical signs are shown by broad horizontal base line. The dotted line suggests the probable course of the temperature had $1 / 100,000$ th milligramme T.R. been administered on Sept. 6th or 7th. In Fig. 2 , on the other hand, the dotted line indicates probable temperature had 1/100,000th milligramme P.T.R. on Nov. 19th been omitted.

FIG. 2.

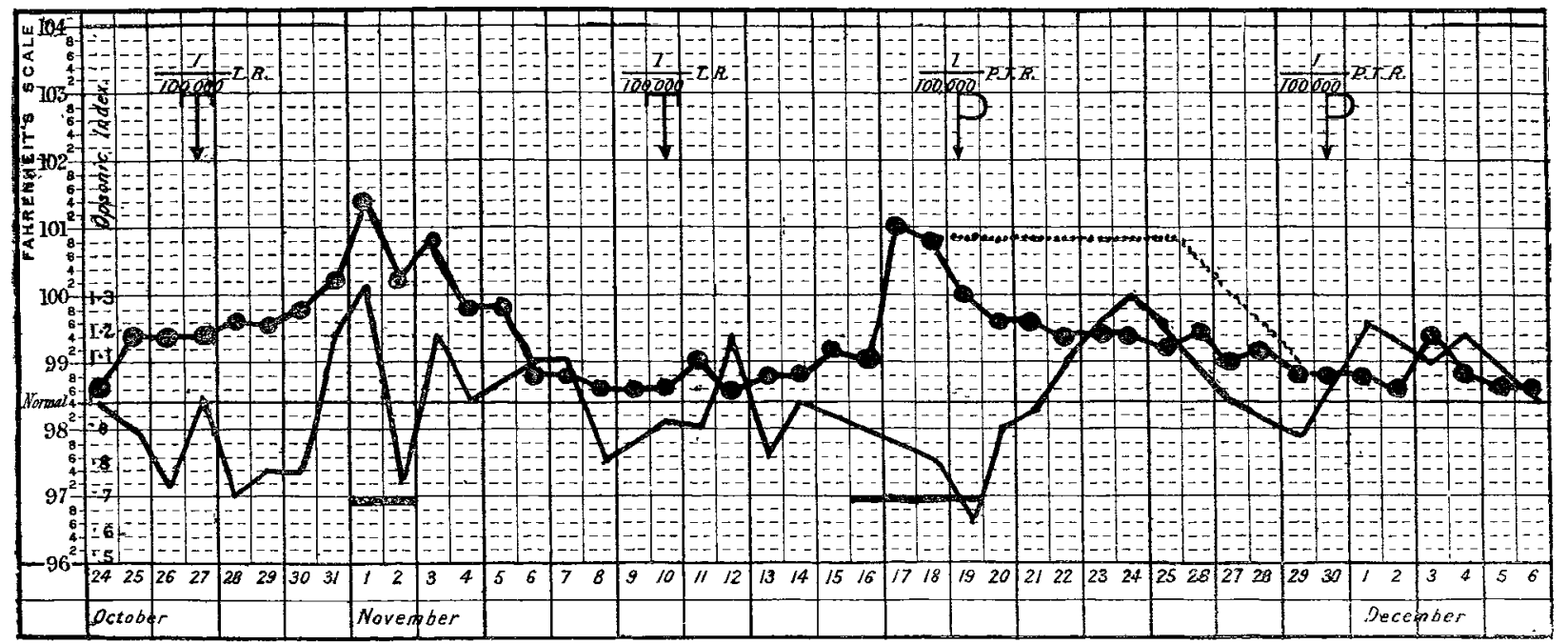

Same patient as in Fig. 1. Further progress of case.

at the lesion. hypothesis.

Without entering further on the various methods in use, and the even more various hypotheses propounded in connexion therewith, I can speak unhesitatingly of the great value of using these two forms of tuberculin in an alternating fashion. Briefly, I have found that a prolonged reaction resulting from the use of either of them can be cut short by a dose of the other provided that there bas been developed some degree of hypersensitiveness to the form of tuberculin which excites the reaction gramme of either T.R. (or P.T.R. in the most acute cases, as it appears slightly less liable than the former to cause acnte reactions). This dose is repeated and gradually increased once every 7 to 10 days until a definite febrile reaction is provoked, accompanied by signs of an exacerbation in the disease, malaise, \&c. The reaction may not appear for three or even six days after an inoculation, hence the interval between the doses. This rise of temperature is allowed to continue until it seems to be steady at the higher level or until the physical condition of the patient causes alarm, when a dose of P.T.R., half the quantity of the exciting dose 
of T.R. (but not as a rule less than 1/100,000th milligramme), is inoculated. The next day the temperature may remain up, but the day after at latest it will fall, and after a few days there will be found to be a definite improvement in the physical condition of the patient and also in the local lesion. This inhibitory dose is the exception above referred to. It is now advisable to rest for a time, say a fortnight or until the temperature has again risen, when the same dose of P.T.R. must be repeated and gradually increased, until for a second time a febrile reaction is induced, when it will be found that a dose of T.R. will now have the effect of cutting short the P.T.R. reaction.

Fig. 1.-Disregarding for the moment the opsonic curve, this chart shows so well a reaction accompanied by high temperature cut short by P.T.R. that I insert it here, although the exciting dose was a mixture of T.R. and P.T.R. I have found the same action of P.T.R. in other cases when I had used mixed tuberculin, but not invariably, and I ascribe it to the actual breed of tubercle bacillus in the lung. Stained by Spengler's method, this patient's sputum never showed any but the human type of tubercle bacillus.

The reason why I used mixed tuberculin is not material to this paper; suffice it to say that I have now discarded it in favour of T.R. and P.T.R. used separately as herein described.

The next dose of P.T.R. had no effect on the temperature, but the third dose, 1/50,000th milligramme, was followed by a definite reaction, though less acute than the former. Had I given a dose of T.R. on Sept. 6th or 7th I feel sure the reaction would have been limited to five or six days instead of ten. The most striking feature in this patient was the very remarkable improvement in the physical signs at the end of September as compared with the end of July. Improvement in general health one can obtain by open air, rest, feeding, and gentle exercise, but not such rapid improvement in physical signs.

Fig. 2.-Discussion of the opsonic curve is again reserved. This chart shows the same scheme of dosage continued. The patient is the same as in Fig. 1 . A period of six weeks was allowed to pass after the last reaction before inoculations of tuberculin were resumed. During this "off time" the health improved, the tem. perature was but seldom above $99^{\circ} \mathrm{F}$., the patient was walking daily, but the physical signs remained stationary. I have omitted, for the sake of clearness, certain doses of second organism vaccines, given sometimes with the tuberculin, sometimes alone. Their effect was difficult to adjudge, but, on the whole, the resulting tendency was to a lower range of temperature and less sputum. Microscopically there was to be seen a numerical reduction of the secondary organisms in the sputum. On Dec. 9th a third dose of bovine tuberculin (1/100,000th milligramme) was given and was followed by a distinct though mild reaction (highest evening temperature $100 \cdot 2^{\circ}$ ). A certain hypersensitiveness had clearly developed as a result of the repeated inoculations with P.T.R.

The following notes of a somewhat more chronic case (evening temperature in reactions $101^{\circ} \mathrm{F}$. maximum, at other times ranging between $98.6^{\circ}$ and $100^{\circ}$ ) provide a more extended view of the treatment.

\begin{tabular}{|c|c|c|}
\hline Date. & Dose. & Remarks. \\
\hline 1909. & & \\
\hline Sept. 3rd & 1/100,000th milligramme P.T.R. & No reaction. \\
\hline$" 8$ th & $1 / 50,000$ th & Slight reaction. \\
\hline " 20th & $1 / 100,000$ th & No reaction. \\
\hline$\because 28$ th & $1 / 50,000$ th & $\begin{array}{l}\text { Marked reaction con- } \\
\text { tinued until the next } \\
\text { dose. }\end{array}$ \\
\hline Oct. 5 th & 1/100,000th milligramme T.R. & Temperature fell. \\
\hline , 28th & $1 / 100,000$ th & No reaction. \\
\hline Nov. 5 th & $1 / 50,000$ th & Short but sharp reaction. \\
\hline "11th & 1/100,000th milligramme P.T.R. & No reaction. \\
\hline , 30th & $1 / 100,000$ th & Very slight reaction. \\
\hline Dec. 7 th & $1 / 50,000 \mathrm{th}$ & Short but sharp reaction. \\
\hline , 17 th & 1/100,000th milligramme T.R. & No reaction. \\
\hline
\end{tabular}

The inoculations were accompanied by steady improvement. It will be noted that after a marked reaction, even though short, a change in the vaccine is made.
It is plain from a consideration of Figs. 1 and 2, and the notes of the chronic case, that after a series of inoculations of one form of tuberculin-T.R. or P.T.R. as the case may be a reaction develops as the result of hypersensitiveness, and that this reaction may be terminated by an inoculation of the other form. These two central facts will, I am convinced, be amply confirmed on extended trial; but whether the doses I have indicated are the most efficient, either during the sensitising process or for cutting short the reaction, I prefer to leave sub judioe. I certainly would hope that my present practice, as set forth above, may be gradually improved upon, though I believe that if rigidly adhered to it will be found to embody a greater measure of safety than has as yet been obtainable.

Before touching on any theoretical consideration I would first ask the medical reader, whether he specialises in the treatment of lung disease or whether he may hitherto have hesitated to embark on the hazardous course of treat. ment by tuberculin in acute phthisis, to dwell once more on the extreme importance of the power which he possesses of cutting short a reaction arising unexpectedly during a course of inoculations. To be able to allay the natural anxiety of his patient during a seemingly acute exacerbation by prophesying the date when the symptoms will abate is to create a sense of security and confidence on both sides which can only make for mutual benefit. And if as I believe, each such carefully limited reaction will bring the nltimate goal of complete cure nearer, then the value of tuberculin, accompanied as it must be in acute phthisis by fluctuations of temperature, will be most amply demonstrated.

\section{Theoretical Considerations.}

I would now advance three propositions, important from a practical standpoint in that, if accepted, they provide a firm base on which the inoculator can take his stand, and whence he can follow whatever hypothesis attracts him into the labyrinth of speculation surrounding the phenomena of active immunisation.

1. Auto-inoculations theoretically must have a greater curative action than therapeutic inoenlations of tuberoulin in the treatment of phthisis (whtther acute or ofronic).-I am using this word auto-inoculations in the generally accepted sense-that is, as connoting the sum total of products, poisonous to the human organism, including quite probably living germs, which by any means whatever are made to leave the focus of infection and enter the general blood or lymphatic systems. It is certain that a stream of such products must always be entering the circulation from an active tubercular focus in the lung, and so in a sense autoinoculation is always proceeding; but from time to time something more than this occurs, and the result is a definite recognisable fluctuation in the patient's condition, comparable to the negative and positive phases (Wright) of a therapentic inoculation curve. This last is the picture I wish to bring before the mind when using the term auto-inoculation.

It will be granted that the more closely a vacine conforms in its constitutior to the bacterial products produced in the lesion, the more efficient will be the action of that vaccine, for this is the basis of the inoculator's art. Obviously one can never hope to produce artificially such a vaccine so perfectly mixed and graded as would stimulate the production by the immunising mechanism of the exact proportion of antibodies required to neutralise respectively bacterial poisons, elaborated by the tubercle bacillus and possibly several secondary organisms, and poisons resulting from cell destruction. Auto-inoculations may not be safe, they may be most dangerous, but theoretically they provide a more perfect vaccine than tuberculin even combined with other vaccines.

Space will not permit me to do more than mention Paterson's magnificent results at Frimley through the systematic adoption of auto-inoculation in afebrile cases, or Hort's suggestion to use a spirometer for the production of auto-inoculation when fever prevents exercise. Both these observers are undoubtedly firm believers in the correctness of my first proposition.

2. Tuberoulin treatment causes hypercmia at the lesion.This is generally accepted; I would refer the reader to Roepke and Bandelier ("Tuberculin in Diagnosis and Treatment"). Now Wright, Freeman, and many others have proved that hyperæmia is a cause of auto-inoculation. The more vascular a tissue in which a lesion is situated the more easily 
will hyperæmia result in auto-inoculation. Lung tissue is par exoellence the site where auto-inoculation could most easily be induced by a condition of hyperæmia. My third proposition may therefore in theory be accepted with confidence.

3. Inooulations of tuberoulin cause auto-inooulation. ${ }^{2}$-This proposition leads us on to somewhat new ground. It certainly suggests a new conception. It would be necessary to modify the dictum, "A therapeutic inoculation must be safer than an auto-inoculation, because the former is a measured dose of dead germs and the latter an unmeasured dose of possibly live ones !" As a matter of fact, the amount of autoinoculation which follows a dose of tuberculin does very greatly depend on the dose administered, and since this can be varied to some extent we can control the process; but it also depends, as a few moments of consideration will convince us, on the area of lung which is diseased, and again

FIG. 3.

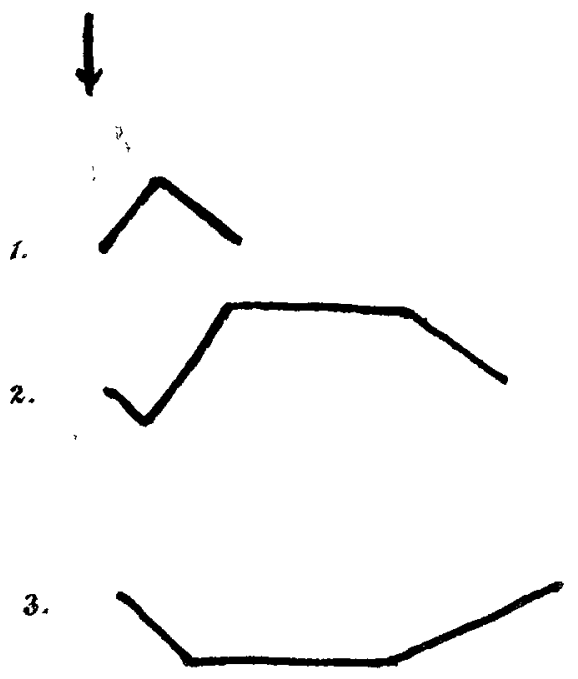

on the sensitiveness of the organism to tuberculin-a sensitiveness which is gradually developed through repetition of equal doses. It will be agreed that if auto-inoculations are an advantage in the treatment of febrile phthisis, as they undoubtedly are in chronic, and if tuberculin can cause auto-inoculation, then tuberculin would be a most convenient method of administering the necessary stimulus. Proposition 3 , then, seems of some importance. I therefore propose to examine what evidence can be found to bear on the subject.
We should expect to find that until the effect of a therapeutic dose of tuberculin had entirely disappeared the clinical and bacteriological picture could be interpreted rationally by supposing the existence of a double wave of immunity instead of a single one-a wave due to tuberculin, followed sooner or later by a wave due to auto-inoculation, a primary wave, and a secondary wave. Since the amount of hyperæmia depends to some extent on the size of the dose-it comes on quicker and is more intense the larger the dose--we should expect to find that the larger the dose the more rapidly would the secondary wave appear, and the more quickly would it obscure the proper course of the primary ware. It is plain then that, except after minute doses of tuberculin, with an immunity curve (Wright) lasting only a few days, could the primary and secondary curve be separate and distinct, as a rule a superimposition of the latter on the former would take place. The primary and secondary waves

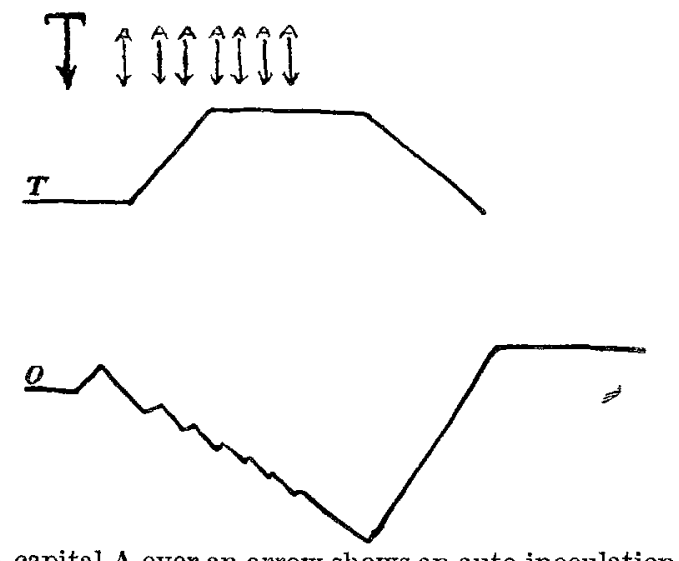

A capital A over an arrow shows an auto-inoculation.

should appear both in the clinical picture and also in the immunity curve as defined by the opsonic index. It will be agreed that the secondary wave is likely in active febrile phthisis to be a very great deal larger than the primary wave, and more especially so when we are dealing with minute doses of tuberculin. We should not be surprised to find that the primary wave clinically was only just recognisable; the secondary, on the other hand, strongly marked, whilst the immunity curve of the primary and secondary waves might approximate respectively to Wright's first and last schematic figures (Fig. 3).

Hitherto, for simplicity, we have regarded - an autoinoculation as a single definite occurrence, like an inocula-

FIG. 5.

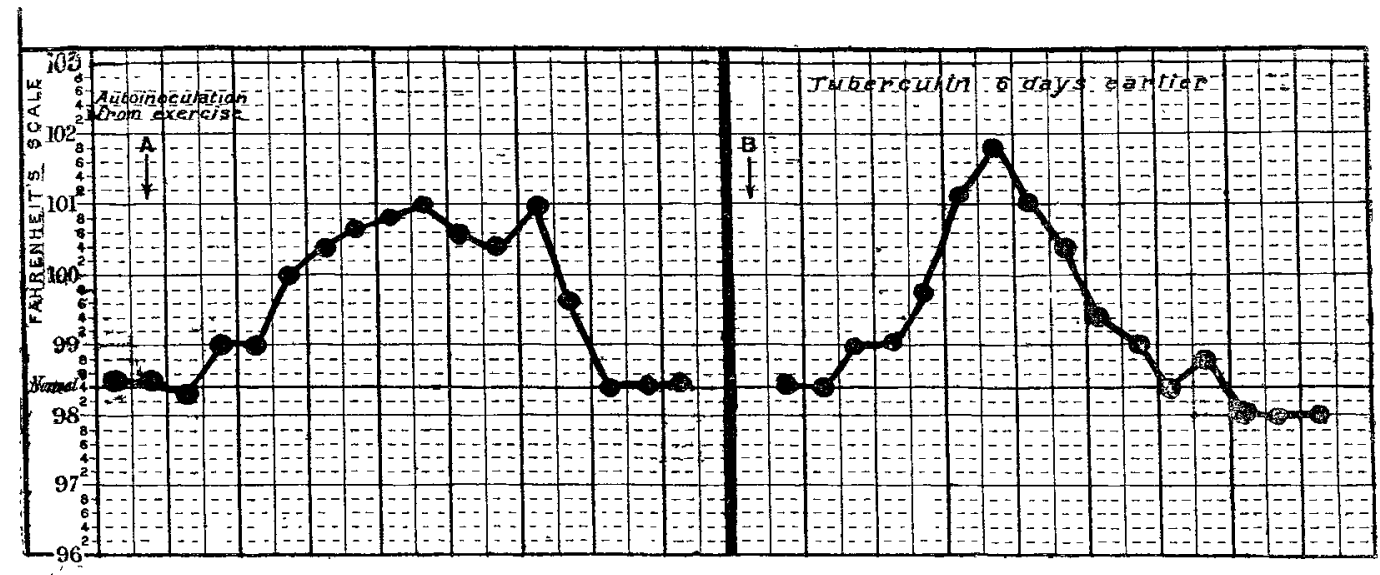

Shows the similarity between an auto-inoculation curve due to exercise and one due to hyperæmia set up by tuberculin.

Let us first, for the sake of argument, assume its correctness-then let us consider what we should expect to find happening as the result of a dose of tuberculin. Lastly, let us examine actual results and see how they compare with the theoretical.

2 Note that in these propositions (2 and 3) I am careful to avoid dogmatising on the effect of one inoculation of tuberculin, for it mia wen the doses are small. tion of tuberculin. Obviously this is not a correct view ; it should be regarded rather as a rapid series of inoculations, small or larger, as the case may be, forming one continuous whole. Taking the temperature as a guide to the clinical condition and the opsonic curve to represent the immunity we might expect to find something of this kind (Fig. 4).

Again, we ought to find a close resemblance between the secondary wave (when separated from the primary) and an auto-inoculation the result of excessive exertion (Fig. 5) 
Finally, it might be supposed that if during a prolonged inoculations induced by the hyperæmia. I will not further negative phase occurring as the result of the secondary wave a enlarge on the point, save by suggesting the possibility minimal dose of tuberculin could be given, which would not that this principle may be found to underlie many of further increase the hyperæmia, then a positive phase might be immediately brought on.

Let us now see how far the facts compare with the theoretical deductions.

Fig. 6 shows the effect of tuberculin in three different degrees. Schematically we may represent them as shown in Figs. $7 \mathrm{~A}, 7 \mathrm{~B}$, and $7 \mathrm{c}$.

In $\mathrm{A}$ the primary wave is very short, and is not recognisable clinically ; then follows the negative phase of the secondary wave.

FIG. 6.

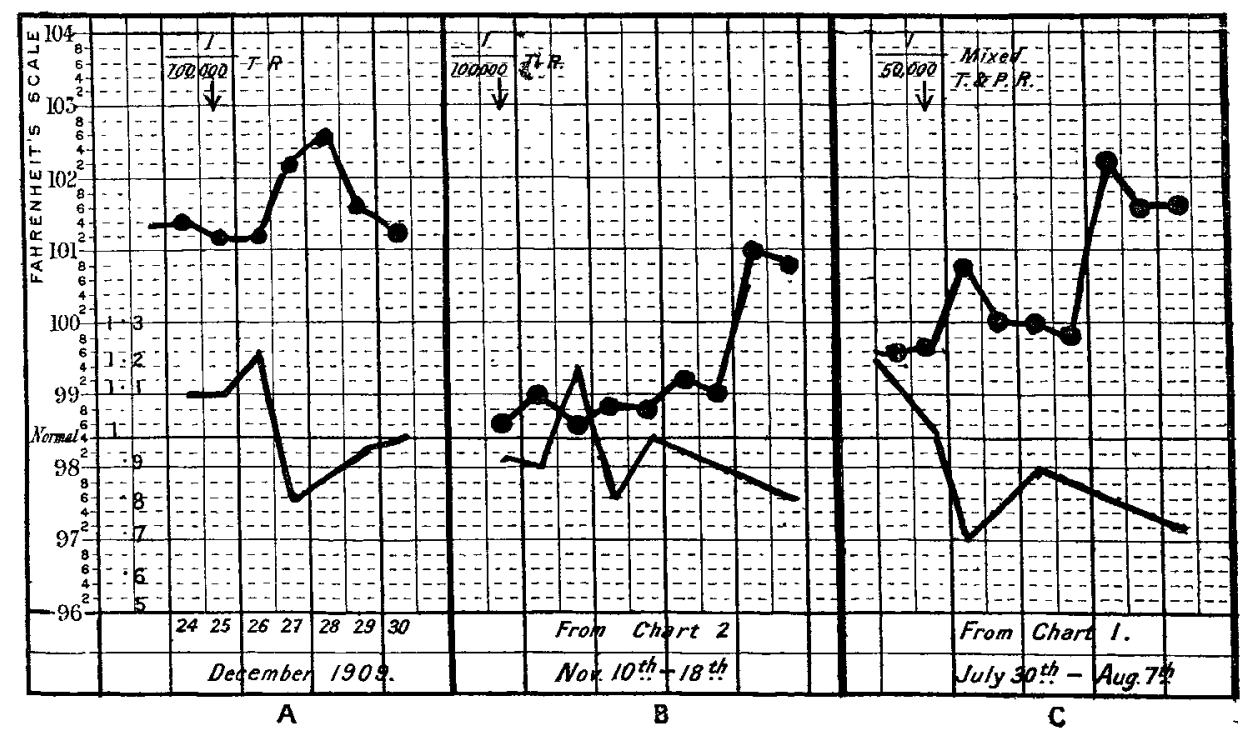

Showing effect of tuberculin in three different degrees. the phenomena associated with the exploitation of vaccines other than tuberculin.

There still remain the questions: (1) of the causation of the hyperæmia; (2) of the development of hypersensibility or "anaphylaxis"; and (3) of the inhibitory action of a different form of tuberculin.

Many theories have been advanced to account for this hyperæmia, none entirely satisfactory. For details I would refer the reader to Emery's “Immunity and Specific Therapy." I cannot but think that the authors of these various explanations taken from Fig. 2, Nov. 10-18, there is a more fully developed primary wave also complete. As the immunity curve begins to rise after the drop at the end of the positive phase it is suddenly checked by the advent of the negative phase of the secondary wave. Turn for one moment to Fig. 2, Nov. 17th et seq., and see the further development of this wave; it continues to show a prolonged negative phase until a dose of bovine tuberculin given on the 19th apparently brings about a typical positive phase. It is probable that the bovine tuberculin did not cause any increased hyperæmia.

In C, taken from Fig. 1, July 30th to August 7th, the primary wave is only just commencing; it is, in fact, only nearing the end of the negative phase; then the negative phase of the secondary wave is suddenly superimposed. Clinically the picture is equally plain : first two days of malaise following the dose, then three days of amelioration ; lastly, a prolonged and distressing reaction. Here, then, the facts coincide very well with the theory.

Further rather striking confirmation is afforded by the next schema. This represents very closely the chart of a case of afebrile phthisis which I inoculated some two years ago, with doses varying from $1 / 10,000$ th to 1/6500th milligramme T.R. (modern reckoning). The temperature was steadily normal during the course except on two occasions - the first when I omitted a dose whilst away on a holiday and the second some 17 days after the last dose was given (Fig. 8). One can plainly see the primary and secondary waves distinct, the secondary curve is stepped and suggests that auto-inoculation is a process taking time.

If the truth of these propositions be admitted as far as they relate to acute phthisis, it is certainly probable that also in afebrile cases the chief value of tuberculin lies in the auto-

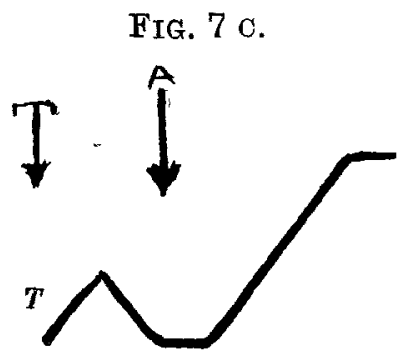

FIG. 7 B.

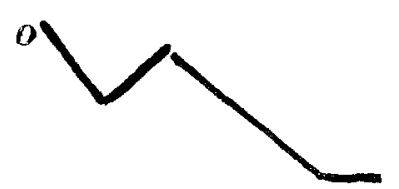

FIG. 8.
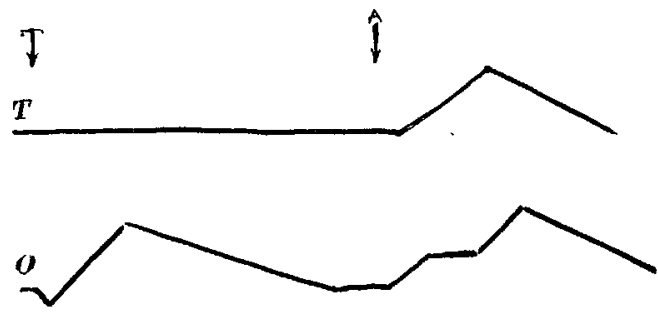
had in their mind the central nervous system as the immediate agent in the production of the hyperæmia, for it is almost incredible that patho. logical hyperæmia should differ in its origin so entirely from physiological hyperæmia as to arise independently altogether of nerve control. But no suggestion seems to have been definitely brought forward as yet that an afferent nerve, a nerve cell, and an efferent nerve are integral and necessary parts of the phenomenon. Yet surely it must be so, and although it may be possible to cut out the afferent nerve by presupposing a direct stimulation of a nerve centre by the circulating poisons in the blood, yet this does not materially affect the position.

Admit the possibility of collaboration by the central nervous system in the production of immunity and the problem certainly becomes simplified. It would not be greatly stretching the imagination to ascribe to the central nervous system the power of causing the elaboration of anti-bodies, whether in response to artificial tuberculin or to autogenous autoinoculation products. The elaboration of $\mathrm{HCl}$, alkalis, most ferments, and various internal secretions are certainly under control of the central nervous system; why not antibodies? But whether one would go to such a length or no, it is more than probable that pathological hypermemia is the result of nerve stimulation.

One would regard a prolonged reaction following tuberculin as a vicious circle wherein, firstly, the central nervous system, stimulated either directly or indirectly through some afferent nerve by the artificial tuberculin, produces a hyperæmia. Secondly, the hyperæmia gives rise to auto-inoculations, of which the products in turn evoke increased hyperæmia, and so on. Whilst all the time nature is striving to produce a sufficiency of antibodies, to combine with all the toxic 
substances formed during this period. At last she is successful and the circle is broken, for the auto-inoculation products become neutralised as soon as they are formed and before they are able to further stimulate the central nervous system. The hyperæmia subsides and the reaction is at an end.

It must be admitted that artificial tuberculin probably differs very considerably in constitution from the product of the tubercle bacillus grown in the human system; hence at its first introduction the central nervous system may not recognise it as akin to the enemy within the lung. Therefore, no stimulus is provided to increase the hyperæmia at the lesion, yet at the same time antibodies must be produced to meet the new element now in the system, and though these antibodies may differ from those already under process of manufacture, yet there may be sufficient similarity to turn the scale against the products of auto-inoculation, and thus we can account for an improvement after the first dose. Exaggerated reactions may follow later doses, as the central nervous system gradually learns to associate tuberculin with the products of the disease which has attacked the system. In like manner, if a savage were to hear the dinner bell for the first time it would convey to him no meaning, but after he found that it preceded a meal, then at the third or fourth time of hearing the welcome sound his central nervous system would have become educated to its significance, and would set in motion the elaborate mechanism of digestion. An application of the same idea is all that is required to explain the action of bovine tuberculin in cutting short an auto-inoculation. Again a different product; to complete the simile it rings a different bell.

Tuberculin, then, would have a two-fold action. It always must excite the production of anti-tuberculin, it sometimes may also stimulate the central nervous system.

To sum up: 1. On general principles auto-inoculations should have a greater effect in influencing the course of phthisis for good than artificial tuberculin, that is, regarding tuberculin merely as an agent which stimulates the system to produce antibodies. 2. But tuberculin itself, if not at first, after repeated doses, causes auto-inoculation. 3. Tuberculin provides an easy method of inducing autoinoculation in acute phthisis. 4. Auto-inoculations are dangerous in acute phthisis if they be too prolonged in their effect. 5. The power to stop an auto-inoculation at will diminishes this danger. 6. By using T.R. and P.T.R. in an alternating fashion, whilst the patient becomes sensitised to the one form, the power to prevent mischief is vested in the other, and on a continuance of inoculations of that other the inhibitory action is transferred to the former. 7. For a full development of this inhibitory action hypersensibility should be developed, hence small doses should be used because an auto-inoculation effect after the first dose or two is not desirable. 8. That the central nervous system may possibly play an important part in the processes of immunisation.

Yelverton, Devon.

\section{THE TREATMENT OF GRAVES'S DISEASE WITH THE MILK OF THYROIDLESS GOATS.}

By WalTer EDMUNDS, M.A., M.B., M.C. Cantab., F.R.C.S. ENG.,

SURGEON TO THE PRINCE OF WALES'S GENERAL HOSPITAL, TOTTENHAM.

A REPORT on this method of treatment was made two years ago ${ }^{1}$ and again one year ago ${ }^{2}$; the present communication continues the previously recorded cases and adds others.

Of Case 1 nothing more has been heard; Case 2 is said to remain in good health; Case 3 is important because severe, and therefore a severe test. During the latter half of 1907 the patient took the milk from a thyroidless goat, and again during the whole of the summer of 1908; throughout 1909 there was given the dried whipped blood from a thyroidless goat, but early in 1910 it was found to cause diarrhoea, and was therefore stopped. During the whole of this time the patient was, as she still is, seriously ill, and was for a considerable period confined to her bed; the goitre is large, the

1 The Lancet, Jan. 25th, 1908, p. 227.

2 The LaNCET, April 10th, 1909, p. 1040 pulse frequent; there are also exophthalmos and occasional attacks of dyspncea at night. There is no evidence that the specific treatment by rodagen, by milk from thyroidless goats, or by dried blood from them was of any benefit in this case. Of Case 4 there is no further information.

CASE 5.-During the early part of the summer of 1909 this patient again had the goat's milk, and in June, 1909, commenced $X$ ray treatment to the thyroid, the applications being at first twice, then once a week. She also took thyroidectin when the milk-supply failed. In November she was again admitted to hospital for a short time. She is now much better ; the eyes are less prominent, she herself is less nervous, and the goitre is much smaller; it can now be distinguished into two separate soft lateral lobes and a central lobe, hard and tender; the diminution in the size of the goitre seems to be due to the $\mathrm{X}$ ray treatment.

of Cases 6, 7, 8, and 9 nothing more has been seen. Case 10 was seen again in October, 1909 ; she is better than she was ; the goitre is not very large, but is still obvious; it is firm to pressure ; the eyes are still prominent.

CASE 11. -Early in 1909 a thyroidless goat was given to this patient, and she took its milk from April to December, 1909. She now considers herself much better in every way than she has been for years; she sleeps better; she is subject to attacks of palpitation about once a fortnight, generally at night; they last about half an hour; she says her heart is much better. The eyes are very little prominent, but the lids do not follow the globe down; the throat is much smaller ; pulse 80 .

Of Cases 12, 13, 14, and 15 there is no further information. The following four cases are additional.

CASE 16.-Male, aged 34 years. The symptoms commenced early in 1908, but the patient did not seek advice until November of that year, when he was found to be suffering from fairly typical Graves's disease. He was treated with rodagen, but he did not improve; indeed, he became worse. He was then given thyroidectin, which he thinks does him good and which he therefore continues to take. In March, 1909, a consultation was held on the case and it was advised that the patient for three months should give up his work and rest in bed, having at the same time the milk of a thyroidless goat. Under this treatment the patient got much better, though he lost weight. After three months he went to the seaside and stopped the goat's milk; a month later he returned and resumed his work. He has now regained his weight and is in good bealth. The throat is of about the same size, but the eyes are no longer markedly prominent.

CASE 17. - A single woman. The symptoms commenced six years ago. In the spring of 1909 she was seriously ill with Graves's disease ; pulse 145, attacks of dyspnoea at night, and a large goitre. She was treated with the milk from a thyroidless goat for four and a half months and also took rodagen. She greatly improved.

CASE 18. - A married woman, aged 28 years. She was admitted to hospital in May, 1909; obvious exophthalmos, pulse 120, thyroid uniformly and distinctly enlarged but not greatly so, tremors in hands. She was treated with milk from thyroidless goats, having from 35 to 40 ounces a day. When she left the hospital in two and a half months' time she was much better. In February, 1910, she was not so well and was readmitted. There is still obvious exophthalmos.

CASE 19. - A single woman, aged 24 years. 'The Graves's disease commenced four or five years ago; 18 months ago it was worse than at present (May, 1909), when this note was taken : "Pulse is 140 ; there is a uniform enlargement of the thyroid gland; there is also slight but distinct exophthalmos; it has been more marked than at present." During the summer of 1909 the patient took the milk of three thyroidless goats which had been operated on for her by her medical man, having for part of the time as much as two quarts a day; by September she felt herself much better; the exophthalmos was not so noticeable as in May and the goitre was smaller.

In the winter of 1908-09 I supplied some thyroidless goats to Dr. J. R. Gilmour of the West Riding Asylum, Scalebor Park, who tried the treatment on cases of Graves's disease under his care on account of mental symptoms, and has pubjished his results. ${ }^{3}$ In two cases there was considerable improvement, and in a third complete recovery. This last

3 The Mental Symptoms in Cases of Exophthalmic Goitre and their Treatment, Journal of Mental Science, October, 1909. 